\title{
BACTERIOLOGICAL PROFILE OF SURGICAL SITE INFECTIONS AND THEIR ANTIMICROBIAL SUSCEPTIBILITY PATTERN AT TERTIARY CARE CENTRE IN KANPUR
}

\author{
Ambika Bhatiani ${ }^{1}$, Vikas Mishra², Nidhi Pal ${ }^{3}$, Ashish Chandna ${ }^{4}$ \\ ${ }^{1}$ Associate Professor, Department of Microbiology, Rama Medical College Hospital and Research Centre, Kanpur, Uttar Pradesh. \\ ${ }_{2}^{2}$ Assistant Professor, Department of Microbiology, Rama Medical College Hospital and Research Centre, Kanpur, Uttar Pradesh. \\ ${ }^{3}$ Ph.D. Scholar, Department of Microbiology, Rama Medical College Hospital and Research Centre, Kanpur, Uttar Pradesh. \\ ${ }_{4}^{\text {nd }}$ Year Postgraduate, Department of Biochemistry, K.B.N.I.M.S, Gulbarga, Karnataka.
}

\begin{tabular}{l} 
ABSTRACT \\
\hline BACKGROUND \\
Healthcare-Associated Infections (HAI) are considered to be the most common and deadliest events menacing the health of \\
patients. Surgical Site Infection (SSI) events are one of the noteworthy healthcare-associated infections and common complication \\
associated with surgery. \\
The aim of this study was to find the incidence of postoperative surgical wound infections in clean, clean-contaminated and \\
contaminated surgeries and to determine bacteriological profile with antimicrobial susceptibility patterns of the isolates.
\end{tabular}

\section{MATERIALS AND METHODS}

During one year of study, total 833 operated cases were included from different departments like orthopaedics, surgery and obstetrics and gynaecology and all the clinical details of the patients were recorded. For microbiological study, swabs were collected from infected surgical sites and processed according to standard procedure.

\section{RESULTS}

The incidence of SSIs in our setting was 1.8 per 100 patients and the rates of infection were highest in contaminated type of surgeries (60\%) followed by clean contaminated (33.3\%) and clean surgeries (6.6\%). Klebsiella pneumoniae (46.6\%) was the most common pathogen followed by Staphylococcus aureus (26.6\%), Pseudomonas species (20\%) and Escherichia coli (6.6\%) isolated from SSIs. Antibiogram of these isolates showed that gram negative bacilli as well as gram positive cocci were resistant to penicillin and cephalosporins. But piperacillin-tazobactam, cefoperazone-sulbactam, imipenem, meropenem, tigecycline and polymyxin drugs were found to be effective. Antimicrobial profile of Staphylococcus aureus showed $75 \%$ and $100 \%$ sensitivity for cotrimoxazole and clindamycin respectively.

\section{CONCLUSION}

The rate of SSIs observed in this study was comparatively lower, but there is higher degree of antimicrobial resistance. It is very important to adhere to strict infection control measures and maintenance of proper hand hygiene.

\section{KEYWORDS}

Antibiogram, Surgical Site Infections.

HOW TO CITE THIS ARTICLE: Bhatiani A, Mishra V, Pal N, et al. Bacteriological profile of surgical site infections and their antimicrobial susceptibility pattern at tertiary care centre in Kanpur. J. Evolution Med. Dent. Sci. 2016;5(82):6141-6144, DOI: 10.14260/Jemds/2016/1387

\section{BACKGROUND \\ Surgical Site Infections (SSIs) constitute a major public health problem worldwide and several studies have shown that SSIs are one of the most common Healthcare-Associated Infections, (HAIs) among hospitalised patients. They are responsible for increasing the cost of treatment, length of hospital stay and significant increase in morbidity and mortality. $[1,2]$}

Financial or Other, Competing Interest: None.

Submission 07-09-2016, Peer Review 01-10-2016,

Acceptance 07-10-2016, Published 13-10-2016.

Corresponding Author:

Dr. Vikas Mishra,

"Geetanjali Villa," 38/157,

Behind PNB, Meston Road,

Kanpur-208001,

Uttar Pradesh,

India.

E-mail: drmishravikas@gmail.com

DOI: $10.14260 /$ jemds/2016/1387
The CDC's Healthcare-Associated Infection (HAI) prevalence survey found that there were an estimated more than one lakh surgical site infections associated with inpatient surgeries in 2011.[3]

Surgery has made great advances in last few decades, but postoperative wound infection is the most common complication faced by surgeons since the advent of surgery. Infection causes delayed recovery and may leave permanent disability. Despite efforts to have a better understanding of sepsis, wound infection is still a clinical problem and some infections in clean wounds remain unexplained. Wound access to bacteria can be prevented by aseptic surgical techniques. ${ }^{[4]}$ The main objective of the present study was to find the incidence of surgical site infections in clean, cleancontaminated and contaminated surgeries in our setting along with the factors related to patient and surgical procedures influencing the rate of postoperative surgical wound infections and to determine bacteriological profile with antimicrobial susceptibility patterns of the isolates. 


\section{MATERIALS AND METHODS}

This was a prospective study conducted in the Department of Microbiology at Rama Medical College Hospital and Research Centre, Kanpur. The study period was from January 2015 to December 2015. Ethical approval was taken from the Institute's Ethical Committee. A total of 833 patients undergoing surgery were taken from different departments like orthopaedics, surgery and obstetrics and gynaecology. All the clinical details of the patients were recorded. Each patient was followed from the time of admission till discharge from the hospital (CDC, 1999). ${ }^{[5]}$

\section{Inclusion Criteria}

All the patients undergoing any type of surgery, clean surgeries (Class I Operative Wounds), clean-contaminated surgeries (Class II Operative Wounds), contaminated surgeries (Class III Operative Wounds) according to CDC classification were included in this study.

\section{Exclusion Criteria}

All type of eye surgeries was excluded. Dirty surgeries (Class IV operative wounds) and contaminated samples were also excluded from the study.

\section{Sample Collection}

Serous or non-purulent or pus discharge was collected aseptically in sterile syringe or in two sterile swab sticks (one for microscopy and another for culture) from the wound.

\section{Processing}

A smear was prepared from direct sample and stained by Gram-staining method for early presumptive diagnosis and sample inoculated on blood agar and MacConkey's agar. The plates were incubated at $37^{\circ} \mathrm{C}$ overnight for $18-24$ hours. After incubation culture plates were observed and isolated organism was identified by colony characteristics and appropriate biochemical reactions. Antimicrobial susceptibility testing was done using disk diffusion tests according to CLSI guidelines. ${ }^{[6]}$

\section{RESULTS}

Among 833 operated cases, 15 suffered from surgical site infection. The incidence of infection was 1.8 per 100 patients. Male and female ratio was 1.5:1. Among 15 infected cases most of postoperative infectious cases were $>50$ years $(66.6 \%)$. [Fig-1] The rate of infection was highest in contaminated type of wounds $60.0 \%$ followed by clean-contaminated wounds $33.3 \%$ and least in clean wounds $6.6 \%$ [Fig-2].

\section{Fig 1: Agewise distribution}

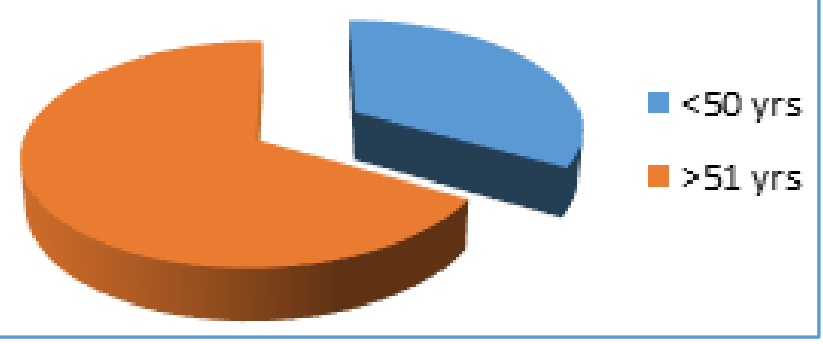

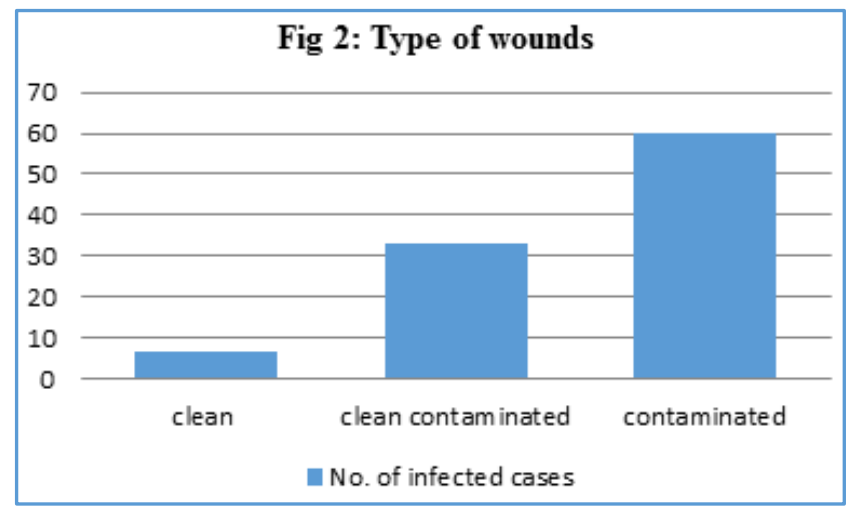

Klebsiella pneumoniae (46.6\%) was most common pathogen isolated from SSIs followed by Staphylococcus aureus (26.6\%), Pseudomonas species (20\%) and Escherichia coli $(6.6 \%)$ [Fig-3].

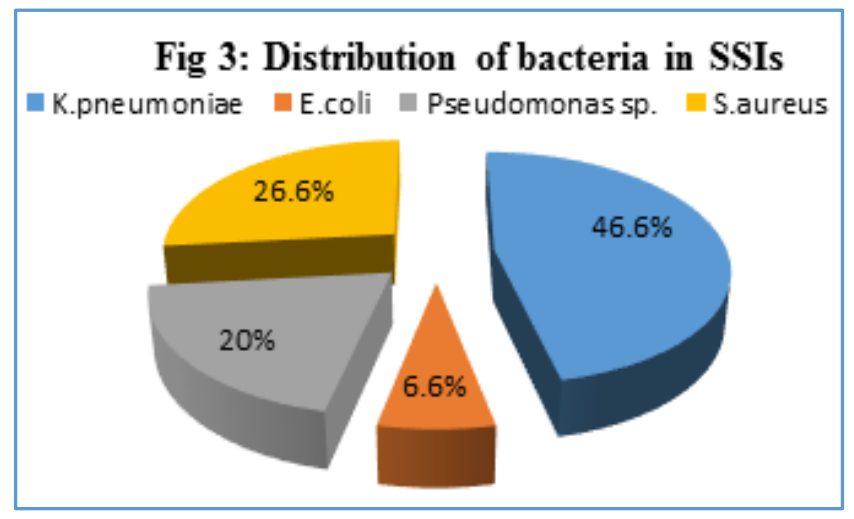

Antibiotic susceptibility testing showed that maximum isolated strains of gram negative bacilli as well as gram positive cocci were resistant to penicillin and cephalosporins. But combination with inhibitors like piperacillin-tazobactam and cefoperazone-sulbactam increased the sensitivity for Klebsiella pneumoniae (71.4\%), Pseudomonas species $(100 \%)$ and Escherichia coli (100\%). Only Klebsiella pneumoniae showed sensitivity to aminoglycosides: gentamycin (42.8\%) and amikacin $(28.5 \%)$ while other gram negative bacilli; Pseudomonas species and Escherichia coli were resistant to aminoglycosides. Carbapenems and polymyxins also showed excellent sensitivity against gram negative bacilli [Fig-4]. Fifty percent of Staphylococcus aureus ( 2 out of 4 ) were methicillin resistant, but sensitive to cotrimoxazole (75\%) and clindamycin (100\%).

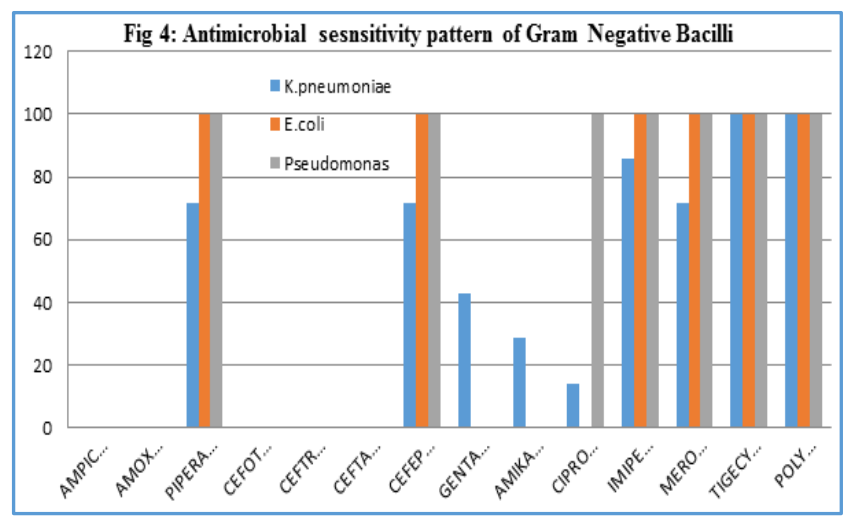




\section{DISCUSSION}

The problem of surgical site infections is seen in both developed and developing countries, despite introduction of strict antiseptic regimes in surgical practices. In this study, 15 patients suffered from surgical site infections. This is comparable with the rates reported by various authors; Lilani et al Wondemagegn Mulu, Ahmed MI and Mundhada AS found SSIs $8.95 \%, 10.9 \%, 25.23 \%$ and $32 \%$ respectively. $[4,7,8,9]$ It can occur from either an endogenous or an exogenous source. As compared to these studies, in the present study infection rate was low which may be due to differences in working conditions or septic conditions or preoperative antibiotic prophylaxis.

There was no significant relation of SSIs with gender as has been reported by other authors also, but age was found to be a significant risk factor for SSIs. In this study patients more than 50 years of age got infected, which was in contrast to other studies.[10]

In the present study surgical site infection was significantly associated with contaminated class of wounds, being the highest (60\%) for contaminated surgery, 33.3\% for clean-contaminated surgery and $6.6 \%$ for clean surgery. While Mundhada AS[4] documented $17.65 \%$ clean and $39.39 \%$ clean contaminated wound were infected and Lilani et al[7] found $3.03 \%$ clean and $22.41 \%$ were clean-contaminated surgeries. This rate of infection would be probably because of profound influence of endogenous contamination during surgery.

Gram negative bacilli were the most frequent pathogen isolated from SSIs in this study and Klebsiella pneumoniae $(46.6 \%)$ was the commonest pathogen followed by Gram positive cocci; Staphylococcus aureus (26.6\%). Masaadeh HA et al[11] and Lilani SP[7] found Pseudomonas sp. as commonest isolate, while Vikrant Negi et al[1] reported E. coli which remains a third and fourth most isolated strain in this study. Fahad A Al-Mulhim[12] and Mundhada AS[4] found S. auerus as the predominant pathogen. Klebsiella, Escherichia coli or any other enteric microorganism infection at a surgical site indicates poor hospital hygiene.

Antimicrobial susceptibility pattern of isolated microorganisms revealed a high degree of resistance. All gram negative bacilli were resistant to penicillin and cephalosporins. Other studies have also shown approximately the same results.[1,11]

On the other hand aminoglycosides, amikacin and gentamycin showed moderate sensitivity against Klebsiella pneumoniae only, other gram negative bacilli were resistant while another study revealed Enterobacteriaceae showing highest sensitivity to amikacin and gentamycin.[13]

Similar to other studies ${ }^{[8,9,11]}$ in the present study Klebsiella pneumoniae, Pseudomonas sp. and Escherichia coli showed good sensitivity to piperacillin-sulbactam $(71.4 \%$, $100 \%$ and $100 \%$, respectively), Imipenem $(85.7 \%, 100 \%$ and $100 \%$ respectively), meropenem $(71.4 \%, 100 \%$ and $100 \%)$ and polymyxin and tigecycline (100\% for all). This could be because these antibiotics are not commonly used and limited in practice because of their higher cost.

In this study, S. aureus was the single gram positive organism isolated. Special interest in S. aureus SSI is mainly due to emergence of Methicillin Resistant Staphylococcus aureus (MRSA) strains, which is difficult to treat. In the present study $50 \%$ of Staphylococcus aureus were Methicillin resistant; this was in contrast to other studies who have reported $10 \%{ }^{[13]}$ and $15 \%{ }^{[14]}$ resistance only. Staphylococcus aureus showed higher sensitivity to co-trimoxazole (75\%) and clindamycin $(100 \%)$ only.

\section{Limitation of Study}

This study was limited to isolate aerobic bacteria only.

\section{CONCLUSION}

The study gives us insight into the bacterial isolates and their resistance pattern in SSIs in our tertiary care hospital. The rate of SSIs in our setting was lower, but there is tremendous resistance of antimicrobial agents. Hence, it is very important to adhere to strict infection control measures, maintenance of proper hand hygiene and judicious use of antibiotics along with evidence-based medicine practice.

\section{REFERENCES}

1. Negi V, Pal S, Juyal D, et al. Bacteriological profile of surgical site infections and their antibiogram: a study from resource constrained rural setting of Uttarakhand state, India. J Clin Diagn Res 2015;9(10):DC17-DC20.

2. Magill SS, Hellinger W, Cohen J, et al. Prevalence of healthcare-associated infections in acute care hospitals in Jacksonville, Florida. Infection Control Hospital Epidemiology 2012;33(3):283-91.

3. Magill SS, Edwards JR, Bamberg W, et al. Multistate pointprevalence survey of health care-associated infections. New England Journal of Medicine. 2014;370(13):11981208.

4. Mundhada AS, Tenpe S. A study of organisms causing surgical site infections and their antimicrobial susceptibility in a tertiary care government Hospital. Indian J Pathol Microbiol 2015;58(2):195-200.

5. Mangram AJ, Horan TC, Pearson ML, et al. Guideline for prevention of surgical site infection, 1999. Hospital infection control practices advisory committee. Infection Control Hospital Epidemiology 1999;20(4):250-78, quiz 279-80.

6. Performance Standards for Antimicrobial Disc Susceptibility Tests. Approved Standard- M100S, 26th ed. Clinical and Laboratory Standards Institute (CLSI), USA. 2016.

7. Lilani SP, Jangale N, Chowdhary A, et al. Surgical site infection in clean and clean-contaminated cases. Indian J Med Microbiol 2005;23(4):249-52.

8. Ahmed ML. Prevalence of nosocomial wound infection among postoperative patients and antibiotics patterns at teaching hospital in Sudan. N Am J Med Sci 2012;4(1):2934.

9. Mulu W, Kibru G, Beyene G, et al. Postoperative nosocomial infections and antimicrobial resistance pattern of bacteria isolates among patients admitted at felege hiwot referral hospital, Bahirdar, Ethiopia. Ethiop J Health Sci 2012;22(1):7-18.

10. Nwankwo EO, Ibeh IN, Enabulele OI. Incidence and Risk Factors of Surgical Site Infection in a Tertiary Health Institution in Kano, Northwestern, Nigeria. 2012. http:// www.ijic.info/article/download/10573/7621.

11. Masaadeh HA, Jaran AS. Incident of Pseudomonas aeruginosa in post-operative wound infection. Am J Infect Dis 2009;5(1):1-6. 
12. Al-Mulhim FA, Baragbah MA, Sadat-Ali M, et al. Prevalence of surgical site infection in orthopedic surgery: a 5-year nalysis. Int Surg 2014;99(3):264-8.

13. Shahane V, Bhawal S, Lele U. Surgical site infections: a one year prospective study in a tertiary care center. Int J Health Sci (Qassim) 2012;6(1):79-84.
14. Aggarwal A, Khanna S, Arora U, et al. Correlation of betalactamase production/methicillin resistance and phage pattern of Staphylococcus aureus. Indian J Med Sci 2001;55(5):253-6. 